\title{
Influenza Associated Neurological Diseases in Children
}

\author{
Agam Jain $^{1} \cdot$ Rakesh Lodha ${ }^{1}$
}

Received: 23 September 2020 / Accepted: 23 September 2020 /Published online: 2 October 2020

(C) Dr. K C Chaudhuri Foundation 2020

Influenza typically presents with respiratory symptoms. However, other organs such as gut, liver, and central nervous system may also be affected. The burden of Influenza Associated Neurological Diseases (IAND) is higher in pediatric population as compared to adults [1], with children accounting for $73 \%$ and $84 \%$ of IAND cases in American [2] and British series [3], respectively. The neurological symptoms typically occur within $14 \mathrm{~d}$ of onset of respiratory symptoms [1]. In a cohort of IAND patients reported in the study by Takia et al. published in October 2020 issue of IJP [4], all patients had fever and respiratory symptoms and presented within 2-7 d of symptom onset.

Neurological symptoms range from altered sensorium, seizures, focal deficit, and represent heterogenous conditions. Some of them could be attributed to febrile seizures, worsening of pre-existing neurological illness secondary to acute illness, hypoxia, sepsis, multi-organ dysfunction but some patients present with neurological involvement in their absence. Among the primary neurological conditions associated with influenza, perhaps the most sinister is Influenza-associated Encephalitis (IAE). IAE represents a wide spectrum of diseases, which are probably a result of aberrant host immune response, with cytokine storm leading to various patterns of injury. There are several patterns of brain injury, most recognized being Acute Necrotizing Encephalitis (ANE). Other patterns include Hemorrhagic Shock and Encephalopathy Syndrome (HSES), Mild encephalopathy with Reversible Splenial Lesions (MERS), Acute Encephalopathy with seizures and late diffusion Restriction (AESD), Encephalopathy with Malignant Brain Edema (EMBE) among others [1]. In the study by Takia et al. [4], of 6 children with IAND, 2 were labelled as ANE, 1 as ADEM and rest as encephalopathy.

Rakesh Lodha

rakesh_lodha@hotmail.com

1 Department of Pediatrics, All India Institute of Medical Sciences, New Delhi, India
There are several challenges faced in treating such patients. Earlier stages of encephalopathy are difficult to pick in young children. Standardized tools such as Cornell Assessment for Pediatric Delirium [5] may help in early identification. Risk factors for neurological involvement include age of 2-4 y and underlying neurological disease [6]. Treatment options in IAND are limited to Oseltamivir and supportive care. Role of new antivirals such as Peramivir, Baloxavir in IAND is not defined. Steroids have been recommended in patients of ANE without brainstem lesions [1] and a recent study suggests Tocilizumab [7] as an add-on therapy in ANE. Another evolving aspect is role of biomarkers such as IL-6 and Cytochrome-c for IAND [1].

There are huge gaps in knowledge regarding neurological manifestations of influenza. It is still unknown how one process leads to such myriad presentations or how to best treat them. The true burden of IAND is also unknown. Proportion of IAND, in children with influenza needing hospitalization varies from population to population [1, 8-10], highest being in Japan and Taiwan [1] ranging from $7.6 \%$ in Australia [8] to $33 \%$ in Taiwan [10]. Takia et al. [4] found the prevalence of IAND in their study to be $8.8 \%$. However, these estimates are from cohorts of admitted patients and might not be accurate, as all influenza patients don't get admitted, not all patients with IAND have respiratory symptoms, and mild encephalopathy in children might be overlooked.

Outcome of IAND ranges from return to premorbid status to severe neurological sequelae to death. In the cohort followed by Takia et al. [4], half of the patients reached premorbid status, $1 / 6^{\text {th }}$ died and remaining had sequelae. Vaccination against influenza is the only effective method for prevention of IAND.

The study by Takia et al. [4] is the first pediatric case series from North India. It highlights the varied manifestation of IAND. Further studies should actively screen for neurological compromise in influenza patients as well as evaluate for influenza in patients presenting primarily with neurological symptoms. 


\section{Compliance with Ethical Standards}

Conflict of Interest None.

\section{References}

1. Tsai JP, Baker AJ. Influenza-associated neurological complications. Neurocrit Care. 2013;18:118-30.

2. Glaser CA, Winter K, DuBray K, et al. A population-based study of neurologic manifestations of severe influenza $\mathrm{A}(\mathrm{H} 1 \mathrm{~N} 1)$ pdm09 in California. Clin Infect Dis. 2012;55:514-20.

3. Goenka A, Michael BD, Ledger E, et al. Neurological manifestations of influenza infection in children and adults: results of a National British Surveillance Study. Clin Infect Dis. 2014;58: 775-84.

4. Takia L, Saini L, Keshavan S, et al. Neurological manifestations of influenza A (H1N1): clinical features, intensive care needs, and outcome. Indian J Pediatr. 2020. https://doi.org/10.1007/s12098020-03297-w.

5. Traube C, Silver G, Kearney J, et al. Cornell assessment of pediatric delirium: a valid, rapid, observational tool for screening delirium in the PICU. Crit Care Med. 2014;42:656-63.
6. Newland JG, Laurich VM, Rosenquist AW, et al. Neurologic complications in children hospitalized with influenza: characteristics, incidence, and risk factors. J Pediatr. 2007;150:306-10.

7. Koh JC, Murugasu A, Krishnappa J, Thomas T. Favorable outcomes with early interleukin 6 receptor blockade in severe acute necrotizing encephalopathy of childhood. Pediatr Neurol. 2019;98: $80-4$.

8. Britton PN, Blyth CC, Macartney K, et al. The spectrum and burden of influenza-associated neurological disease in children: combined encephalitis and influenza sentinel site surveillance from Australia, 2013-2015. Clin Infect Dis. 2017;65:653-60.

9. Mastrolia MV, Rubino C, Resti M, Trapani S, Galli L. Characteristics and outcome of influenza-associated encephalopathy/encephalitis among children in a tertiary pediatric hospital in Italy, 2017-2019. BMC Infect Dis. 2019;19:1012.

10. Chen L-W, Teng C-K, Tsai Y-S, et al. Influenza-associated neurological complications during 2014-2017 in Taiwan. Brain Dev. 2018;40:799-806.

Publisher's Note Springer Nature remains neutral with regard to jurisdictional claims in published maps and institutional affiliations. 